\section{Efecto de la incorporación de registros adicionales a la presión arterial en la Encuesta Nacional de Salud, Chile 2010}

\author{
JOAQUÍN MONTERO ${ }^{1}$, CRISTIÁN MANSILLA², PAULA MARGOZZINI ${ }^{3}$
}

\section{Effects of incorporating additional blood pressure measurements during The National Health Survey in Chile}

Background: It is of utmost importance to identify hypertensive subjects in a country, in order to use efficiently public resources. The National Health Survey 2009-10 in Chile incorporated a third measurement of blood pressure $(B P) d u$ ring the home visit performed by a nurse, and a second day of measurement in a representative sub-sample. Aim: To study the effect of these two additional actions over both the average value of BP and the national prevalence of hypertension. Material and Methods: A third blood pressure measurement was carried out in 5,058 subjects, and it was measured in a second day in 930 individuals. The effect of these additional measurements on absolute blood pressure values and the prevalence of hypertension were assessed. Results: A small but statistically significant reduction in mean systolic pressure $(0.52 \mathrm{mmHg})$ and the prevalence of hypertension (1\%) was observed after the incorporation of the third blood pressure measurement. No effects in these figures were observed after the measurement performed on a second day. Conclusions: These findings should be considered when designing the new National Health Survey in Chile.

(Rev Med Chile 2016; 144: 285-290)

Key words: Blood Pressure; Health Surveys; Hypertension.

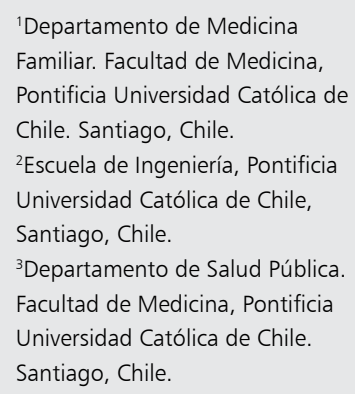

Recibido el 5 de noviembre de 2015, aceptado el 1 de diciembre de 2015.

$$
\begin{aligned}
& \text { Correspondencia a: } \\
& \text { Cristián Mansilla } \\
& \text { Lira63, Santiago. } \\
& \text { camansil@gmail.com }
\end{aligned}
$$

\section{I} a hipertensión arterial (HTA) no sólo es la condición responsable de la mayor carga de enfermedad en Chile (expresado en años de vida ajustados por discapacidad (AVISA)) ${ }^{1}$, sino también es el principal factor de riesgo cardiovascular a nivel mundial ${ }^{2}$. La forma tradicional de detectar esta enfermedad crónica no siempre contempla los valores reales de presión arterial (PA), principalmente por la variabilidad intra-individual que se observa en su medición ${ }^{3,4}$.

Por otro lado, la correlación de la PA medida clínicamente en consulta con el promedio obtenido mediante monitorización ambulatoria de la presión arterial (MAPA) es más bien pobre, siendo pocas veces mayor que $0,5^{5-7}$.

Para abordar la variabilidad intra-individual de la PA, la evidencia muestra que el número de determinaciones que se lleve a cabo tiene un impacto significativo sobre un diagnóstico correcto de $\mathrm{HTA}^{8,9}$. Más aún, la prevalencia de HTA sobre o sub-diagnosticada por este efecto, tendría un impacto relevante para la salud pública ${ }^{10}$.

Pese a la evidencia ya mencionada, la mayoría de los estudios que exploran el impacto de introducir determinaciones adicionales para reducir la variabilidad intra-individual, lo realizan para determinaciones clínicas, sin abordar el contexto de una encuesta nacional. Por otro lado, algunos autores han puesto especial énfasis en la dificultad para realizar comparaciones con prevalencias entre estudios ${ }^{11}$. En términos poblacionales, generalmente se suelen utilizar los métodos tradicionales de registro de $\mathrm{PA}$, lo cual implica que, al momento de comparar y analizar los datos de HTA en la 
población, deben tomarse en cuenta numerosos factores, tales como el método utilizado, las medidas que se tomaron para mejorar la confiabilidad de sus resultados, los protocolos de registro, las condiciones relacionadas a los individuos examinados y a la persona que registra, el ambiente donde se realiza la medición, el número y el tiempo que transcurre entre las determinaciones.

En la Encuesta Nacional de Salud (ENS) realizada en Chile en el año 2003 se encontró una elevada prevalencia de HTA en la población y algunos expertos consideraron que ésta podría ser atribuida a problemas de registro, considerando que sólo se midió dos veces la PA y en un solo día ${ }^{12}$. Para dilucidar estos aspectos, en la ENS de 200910, se agregó una tercera medición y un segundo día de determinación (realizado como piloto en una de las regiones del país).

De esta forma, conocer el impacto que estas intervenciones tuvieron sobre la ENS 2009-10 es de vital importancia para la planificación de ENS futuras, lo que sería el mayor impacto que los resultados de este estudio podrían generar. Por otro lado, obtener una estimación real de la población hipertensa en el país mejoraría la asignación de recursos a un problema de salud pública que padece más del $30 \%$ de los chilenos ${ }^{13}$.

El objetivo de este trabajo es responder a las siguientes preguntas: 1) Cuánto se modifica el valor promedio de la PA y la prevalencia de HTA de la población al agregar una tercera medición en una visita; 2) Cuánto se modifica el valor promedio de la PA y la prevalencia de HTA en la población al agregar un segundo día de determinación. Ambas preguntas se enmarcan en el contexto de una encuesta nacional.

\section{Material y Método}

\section{Definición de hipertenso}

Se consideró hipertenso a la persona mayor de 14 años que tiene una PA igual o superior a 140/90 $\mathrm{mmHg}$ o que reporta el uso de medicamentos antihipertensivos.

\section{Diseño de la ENS}

La ENS 2009-10 realizó un estudio de prevalencia en hogares con una muestra nacional, probabilística, estratificada y multi-etápica de 5.412 personas de 14 o más años. El protocolo de estudio y consentimientos informados fueron aprobados por el Comité de Ética de la Pontificia Universidad Católica de Chile (PUC) y el Ministerio de Salud ${ }^{13}$. El diseño y metodología del estudio se encuentran descritos en el sitio web del Ministerio de Salud.

De igual forma, en el caso de la Región Metropolitana (RM), la ENS 2009-10 realizó una muestra aleatoria de población general, la cual no contiene sesgo de selección.

\section{Medición de la PA}

Las personas fueron visitadas en sus hogares, en dos oportunidades, en las mañanas. Un encuestador realizó la primera visita donde, junto con aplicar una encuesta general y dejar instrucciones para la segunda visita, en la RM llevó a cabo un primer registro de la PA protocolizado, previo reposo de 5 min medidos por reloj, y con un período de $2 \mathrm{~min}$, también medidos exactos por reloj, entre cada una de las 3 mediciones. Una enfermera entrenada realizó la segunda visita. En ella completó la encuesta, donde se consignó si se le ha realizado el diagnóstico de HTA o está recibiendo medicamentos antihipertensivos y se le controló la PA con el mismo protocolo de la primera visita, en forma posterior a la toma de muestra de orina y previo a la punción venosa. Las personas debían estar en ayunas y recibir su medicación antihipertensiva según horarios habituales.

De esta forma, por razones de conveniencia, la segunda visita en la RM, incluyó la medición de la PA. En cuanto al número de determinaciones, la tercera medición de PA se realizó en todo el país.

Los aparatos fueron probados al iniciar el trabajo de campo y periódicamente durante el transcurso del trabajo de terreno, junto con el recambio estandarizado de baterías. Se utilizó un aparato de presión automático Omron HEM $742^{\circledR}$. Estos equipos cuentan con estudios de validación internacional incluyendo uno realizado en población adolescente en América Latina ${ }^{14}$.

\section{Definición de variables}

Con los datos de la ENS, se estudian las significancias de cuatro variables construidas a partir de la diferencia de dos magnitudes:

1. Variable 1: Representa las diferencias que existen entre el promedio de las tres primeras mediciones y el promedio de las dos primeras mediciones, tanto para la presión arterial sistólica (PAS) como diastólica (PAD). 
2. Variable 2: Representa las diferencias que existen entre la prevalencia de HTA considerando el promedio de las dos primeras mediciones y la prevalencia considerando el promedio de las tres mediciones.

3. Variable 3: Representa las diferencias que existen entre el promedio de las tres mediciones tomadas por la enfermera y el promedio de las tres mediciones tomadas por el encuestador en la población de la RM, tanto para la PAS como PAD.

4. Variable 4: Representa las diferencias que existen entre la prevalencia de HTA considerando el promedio de las tres mediciones tomadas por la enfermera y la prevalencia considerando el promedio de las tres mediciones tomadas por el encuestador en la población de la RM.

\section{Metodología de análisis}

El análisis de las variables se separa según los dos objetivos de la investigación: efecto de la inclusión de una tercera medición de PA y el impacto de una segunda visita o día de medición. Para cada variable definida en cada uno de los objetivos (Variables 1 y 2 en el primer objetivo, y Variables 3 y 4 en el segundo), se evalúa la significancia del valor nacional en el caso de las variables 1 y 2 , y de la RM, en el caso de las variables 3 y 4 con un test $t$ de una muestra, evaluando si el intervalo de confianza al 95\% de cada variable incluye el valor 0 .

Por último, en el caso de la variable 2, los resultados se desagregan por sexo, nivel educacional, zona (urbana o rural) y edad de la población encuestada.

\section{Resultados}

Características de la muestra ENS y sub-muestra RM

Para medir el efecto de la incorporación de una tercera medición se utilizó la muestra de población utilizada en la ENS que fueron 5.058 personas, mientras que para la incorporación de un segundo día se utilizó una sub-muestra de 930 personas (Tabla 1, obtenida del informe final de la ENS 2009-10, accesible en www.minsal.cl).

\section{$1^{\text {er }}$ objetivo: Incorporación de una tercera medición}

El promedio de las tres mediciones de PAS fue aproximadamente $0,52 \mathrm{mmHg}$ menor que el valor del promedio de las dos primeras mediciones, valor que es significativo a nivel nacional (Variable 1). El promedio de la PAS de la población bajó de 126,3 a 125,7 mmHg. La PAD no presentó diferencia significativa de valores a nivel nacional.

Tabla 1. Caracterización de la muestra utilizada en la ENS 2009-10, tanto a nivel nacional (utilizada para medir la incorporación de una tercera medición) como de la Región Metropolitana (utilizada para medir la incorporación de un segundo día de medición).

Nivel educacional bajo: < 8 años de escolaridad. Medio: Entre 8 y 12 años. Alto: Más de 12 años

\begin{tabular}{|c|c|c|c|c|c|c|c|}
\hline \multirow[b]{2}{*}{ Edad } & \multirow[b]{2}{*}{ Nivel educacional } & \multicolumn{3}{|c|}{ Diseño muestral nacional } & \multicolumn{3}{|c|}{ Diseño muestral RM } \\
\hline & & Masculino & Femenino & Total & Masculino & Femenino & Total \\
\hline \multirow{3}{*}{$\begin{array}{l}15-24 \\
\text { años }\end{array}$} & Bajo & 18 & 14 & 32 & 2 & 0 & 2 \\
\hline & Medio & 268 & 319 & 587 & 53 & 68 & 121 \\
\hline & Alto & 77 & 106 & 183 & 14 & 23 & 37 \\
\hline \multirow{3}{*}{$\begin{array}{l}25-44 \\
\text { años }\end{array}$} & Bajo & 61 & 116 & 177 & 10 & 9 & 19 \\
\hline & Medio & 427 & 655 & 1.082 & 76 & 96 & 172 \\
\hline & Alto & 199 & 277 & 476 & 46 & 72 & 118 \\
\hline \multirow{3}{*}{$\begin{array}{l}45-64 \\
\text { años }\end{array}$} & Bajo & 187 & 341 & 528 & 11 & 42 & 53 \\
\hline & Medio & 417 & 535 & 952 & 70 & 105 & 175 \\
\hline & Alto & 109 & 160 & 269 & 25 & 37 & 62 \\
\hline \multirow[t]{3}{*}{$>65$ años } & Bajo & 264 & 419 & 683 & 19 & 55 & 74 \\
\hline & Medio & 102 & 165 & 267 & 18 & 40 & 58 \\
\hline & Alto & 27 & 31 & 58 & 6 & 16 & 22 \\
\hline Total & & 2.156 & 3.138 & 5.294 & 350 & 563 & 913 \\
\hline
\end{tabular}


Por otro lado, la prevalencia de HTA, considerando el promedio de las tres mediciones, presentó en promedio una reducción aproximada de $1 \%$ absoluto de la prevalencia con el promedio de las 2 primeras mediciones, lo que es significativo a nivel nacional (Variable 2). Es decir, la prevalencia de la HTA de la población cayó de 30,9\% a 29,9\%. Ambos análisis se muestran en la Tabla 2.

Al desagregar la Variable 2, se observa lo siguiente (Tabla 3):

- Sexo: La prevalencia de HTA para mujeres fue $1,5 \%$ mayor cuando se considera el promedio de las dos versus tres mediciones. En el caso de los hombres, no se observaron diferencias significativas.

- Nivel educacional: La prevalencia de HTA considerando el promedio de las primeras dos mediciones versus las tres fue $1,2 \%$ superior para el nivel medio y $1,3 \%$ para el nivel alto. El nivel bajo no presentó diferencias significativas.
- Zona geográfica: La prevalencia calculada con el promedio de dos mediciones fue $1,1 \%$ superior versus las tres mediciones para la población urbana. La población rural no presentó diferencias significativas.

- Edad: La prevalencia considerando el promedio de dos mediciones fue $1,7 \%$ mayor versus el promedio de tres mediciones para la población entre 25 y 44 años y 1,2\% mayor para las personas entre 45 y 64 años. Las personas entre 15 y 24 años y mayores de 65 años no presentaron diferencias significativas.

\section{$2^{\circ}$ objetivo: Efecto de agregar una segunda} visita para determinación de la PA

El promedio de las tres mediciones de presión (tanto sistólica como diastólica) en un primer día no presentó diferencias significativas con el promedio de las tres mediciones realizadas en un segundo día (Variable 3). Por otro lado, la preva-

Tabla 2. Cambios al comparar tres mediciones versus dos mediciones (nivel nacional) de valores promedios de PAS (Variable 1) y de prevalencia HTA (Variable 2)

\begin{tabular}{|lcccc|}
\hline Variable & Media & \multicolumn{2}{l|}{$\begin{array}{c}\text { Intervalo de confianza 95\% } \\
\text { Inferior }\end{array}$} & Sumperior \\
\hline Variable 1 [PAS] & $-0,52$ & $-0,64$ & $-0,4$ & 4.937 \\
Variable 2 & 0,01 & 0,003 & 0,016 & 4.958 \\
\hline
\end{tabular}

Tabla 3. Significancia de variable 2, que resta la prevalencia de HTA con dos mediciones versus la prevalencia con tres mediciones, desagregado por Sexo, Nivel Educacional, Zona y Edad

\begin{tabular}{|c|c|c|c|c|}
\hline \multirow[t]{2}{*}{ Desagrupador } & \multirow[t]{2}{*}{ Media } & \multicolumn{2}{|c|}{ Intervalo de Confianza 95\% } & \multirow[t]{2}{*}{ Tamaño muestral } \\
\hline & & Inferior & Superior & \\
\hline \multicolumn{5}{|l|}{ Sexo } \\
\hline Masculino & 0,004 & $-0,04$ & 0,012 & 1.992 \\
\hline Femenino & 0,015 & 0,005 & 0,025 & 2.966 \\
\hline \multicolumn{5}{|l|}{ Nivel educacional } \\
\hline Bajo (< 8 años) & $-0,017$ & $-0,012$ & 0,008 & 1.296 \\
\hline Medio (8-12 años) & 0,012 & 0,004 & 0,02 & 2.646 \\
\hline Alto (> 12 años) & 0,013 & 0,009 & 0,03 & 912 \\
\hline \multicolumn{5}{|l|}{ Zona } \\
\hline Urbano & 0,011 & 0,004 & 0,018 & 4.225 \\
\hline Rural & 0,003 & $-0,009$ & 0,014 & 733 \\
\hline \multicolumn{5}{|l|}{ Edad } \\
\hline 15-24 años & $-0,001$ & $-0,01$ & 0,008 & 726 \\
\hline 25-44 años & 0,017 & 0,003 & 0,03 & 1.629 \\
\hline 45-64 años & 0,012 & 0 & 0,024 & 1.644 \\
\hline$>65$ años & 0,002 & $-0,003$ & 0,007 & 959 \\
\hline
\end{tabular}


Tabla 4. Significancia de variables 3 y 4, que muestran el impacto de la incorporación de un segundo momento en la medición de la PA

\begin{tabular}{|lccc|}
\hline Variable & Media & \multicolumn{2}{c|}{$\begin{array}{l}\text { Intervalo de Confianza 95\% } \\
\text { Inferior }\end{array}$} \\
\hline Variable 3[1] (presión sistólica) & $-5,34$ & $-10,88$ & 0,21 \\
Variable 3[2] (presión diastólica) & $-5,3$ & $-11,05$ & 0,45 \\
Variable 4 & $-0,02$ & $-0,06$ & 0,01 \\
\hline
\end{tabular}

lencia de HTA considerando el promedio de las tres mediciones en un primer día y la prevalencia de las mediciones del segundo día tampoco son significativas (Variable 4). Ambas variables se muestran en la Tabla 4.

\section{Discusión}

$\mathrm{Al}$ observar los resultados obtenidos en este estudio, lo primero que llama la atención es la relativa estabilidad de los valores de la PA entre las mediciones, lo que se puede atribuir al seguimiento de un protocolo riguroso, estandarización de tiempos y días, entrenamiento del personal involucrado y uso de equipos automáticos para medir la PA. Esto contrasta con estudios de Fasce et al. en la VIII Región de Chile, que mostraban una gran variabilidad entre determinaciones ${ }^{15}$. Es muy posible que el gran esfuerzo por estandarizar la determinación de la PA que usó la ENS, así como el uso de equipos automáticos, pueda explicar esta estabilidad.

Dentro de los resultados, se destaca la reducción leve, pero estadísticamente significativa, de los valores de la PAS al agregar una tercera determinación, lo que se concentra en la población femenina, urbana, con mayor a 8 años de nivel educacional y entre 25 y 64 años. El análisis de los datos no permite extraer una hipótesis clara para explicar estas diferencias observadas según las características de la población, por lo que para aclararlo debiera considerarse un estudio adicional.

Por otro lado, es significativa la reducción en un 1 punto porcentual (de 30,9\% a 29,9\%) de la prevalencia de la HTA, lo que implica que más de 100.000 personas que fueron diagnosticadas como hipertensas, por aspectos metodológicos, no debieran ser consideradas como tal.

Contrario a lo esperado, el análisis de los datos no arrojó diferencias significativas (tanto en el valor de la PA como en el de prevalencia de HTA) en la incorporación de un segundo día en la determinación de la PA en los encuestados de la RM. Sin embargo, no es posible descartar que existan otros factores que impiden que se apreciara dicha reducción, tales como la expectativa de una punción venosa para extraer sangre para los exámenes después de la determinación de la PA del segundo día o la presencia de efecto "delantal blanco".

De nuestro conocimiento, este es el primer estudio que analiza en Chile la pertinencia de utilizar una tercera medición para determinar la PA. Si bien existe evidencia que muestra la reducción de la variabilidad de presión al incorporar determinaciones adicionales, este efecto no se ha estudiado formalmente en el contexto de una encuesta nacional.

Por otro lado, la variabilidad encontrada no dependería de la magnitud de la medición, dado que al realizar un análisis Bland-Altman, no se encontraron diferencias sustanciales en valores de PA (tanto sistólica como diastólica) extremos.

Además, el objetivo de este estudio fue hacer patente la variabilidad intra-individual en la medición de la PA en el contexto de una encuesta nacional, y cómo esta variabilidad se reduce al incorporar mediciones adicionales. De esta forma, no es del alcance de esta investigación cuestionar las causas fisiológicas o sociales que podrían originar estas diferencias, entendiendo que la PA posee una variabilidad natural la cual, en el contexto de una encuesta nacional, puede abordarse agregando mediciones adicionales.

De acuerdo al estudio de carga de enfermedad hecho en Chile en el año $2007^{1}$, la HTA es el factor de riesgo con mayor mortalidad atribuible y, junto con el alcohol, el consumo de sal y el sobrepeso son los factores de riesgo que explican la mayor 
cantidad de años de vida perdidos por mortalidad prematura (AVPM) en Chile. Estos hechos reflejan la importancia de los resultados de nuestro estudio, y si bien los cambios son sólo leves numéricamente, son importantes puesto que afectan a un gran número de personas. El poder estimar mejor la carga permite proyectar en forma más precisa los recursos necesarios para su cobertura.

A la luz de estos resultados, parece conveniente agregar una tercera determinación de la PA en las futuras ENS ya que, sin complicar mayormente el registro, mejora la precisión y la hace más comparable con otras encuestas internacionales que si usan esta tercera determinación. Por otro lado, dada la ausencia de una diferencia significativa en la comparación de valores entre una o dos visitas, no parece recomendable agregar una segunda visita para el registro de la PA en una próxima ENS, ya que aumenta innecesariamente el costo y tiempos de capacitación de encuestadores. El tamizaje realizado por la ENS es suficiente para vigilancia epidemiológica. Sin embargo, podría ser interesante en un estudio futuro la incorporación de una sub-muestra MAPA en los pacientes en los que se encuentran valores elevados de la PA en la ENS y precisar mejor el sesgo de sobre-diagnóstico por efecto "delantal blanco".

Agradecimientos. La ENS 2009-10 fue financiada por el Ministerio de Salud del Gobierno de Chile y la Facultad de Medicina de la Pontificia Universidad Católica de Chile. Los equipos adicionales para el presente estudio fueron aportados por el Departamento de Medicina Familiar de la Pontificia Universidad Católica de Chile. Agradecemos a Álvaro Passi la gentileza para realizar análisis estadísticos para verificar la validez de nuestros resultados.

\section{Referencias}

1. Pontificia Universidad Católica de Chile y Ministerio de Salud, 2007. Informe final. Estudio de carga de enfermedad y carga atribuible. Disponible en epi.minsal. cl [Consultado el 12 de diciembre de 2013].

2. Lawes C, Vander Hoorn S \& Rodgers A. Global burden of blood-pressure-related disease, 2011. Lancet 2008; 371 (9623): 1513-8.

3. Verdecchia P, Schillaci G, Borgioni C, Ciucci A, Zampi I, Gattobigio R, et al. White coat hypertension and white coat effect. Similarities and differences. Am J Hypertens
1995; 8 (8): 790-8.

4. Hessel P. Terminal digit preference in blood pressure measurements: effects on epidemiological associations. Int J Epidemiol 1986; 15 (1): 122-5.

5. Mancia G, Omboni S, Ravogli A, Parati G, Zanchetti A. Ambulatory blood pressure monitoring in the evaluation of antihypertensive treatment: additional information from a large data base. Blood Press 1995; 4 (3): 148-56.

6. Mancia G, Sega R, Bravi C, De Vito G, Valagussa F, Cesana G, et al. Ambulatory blood pressure normality: results from the PAMELA study. J Hypertens 1995; 13 (12 Pt 1): 1377-90.

7. Imai Y, Nagai K, Sakuma M, Sakuma H, Nakatsuka H, Satoh H, et al. Ambulatory blood pressure of adults in Ohasama, Japan. Hypertension 1993; 22 (6): 900-12.

8. Vinyoles E, Camafort M, Domenech M, Coca A, Sobrino J. Prevalence of masked uncontrolled hypertension according to the number of office blood pressure measurements. Rev Clin Esp 2015; 215 (8): 425-30.

9. Mallion JM, Genès N, Vaur L, Clerson P, Vaïsse B, Bobrie G, et al. Detection of masked hypertension by home blood pressure measurement: is the number of measurements an important issue? Blood Press Monit 2004; 9 (6): 301-5.

10. Obara T, Ohkubo T, Kikuya M, Asayama K, Metoki $\mathrm{H}$, Inoue $\mathrm{R}$, et al. Prevalence of masked uncontrolled and treated white-coat hypertension defined according to the average of morning and evening home blood pressure value: from the Japan Home versus Office Measurement Evaluation Study. Blood Press Monit 2005; 10 (6): 311-6.

11. Silva L, Orduñez P, Rodríguez M, Robles S. A tool for assessing the usefulness of prevalence studies done for surveillance purposes: the example of hypertension. Rev Panam Salud Publica 2001; 10 (3): 152-60.

12. Ministerio de Salud 2003. Resultados I Encuesta de Salud, Chile 2003. Disponible en epi.minsal.cl [Consultado el 23 de octubre de 2014].

13. Ministerio de Salud 2010. Encuesta Nacional de Salud ENS Chile 2009-2010. Disponible en web.minsal.cl [Consultado el 23 de octubre de 2014].

14. Christofaro D, Fernandes R, Gerage A, Alves M, Polito M, Oliveira A. Validation of the Omron HEM 742 blood pressure monitoring device in adolescents. Arq Bras Cardiol 2009; 92 (1): 10-5.

15. Fasce E. Hipertension arterial: Aspectos epidemiológicos. En: Jalil J, Michea L, Passalaqua W, Tagle R, Editores, Manual Clínico de Hipertensión Arterial. Santiago, Chile: Editorial Sociedad Chilena de Hipertensión; 2013 p. 8-13. 